\title{
Embarazo en cicatriz de cesárea previa: ¿Es siempre quirúrgico? Reporte de un caso
}

\section{Cesarean Scar Pregnancy: Always surgical? A Case Report}

\author{
Alessandra Cassana ${ }^{1, a}$, Omar Yanque $2, b$ \\ 1 Departamento de Cirugia General, Hospital Nacional Edgardo Rebagliati Martins, Lima, Perú. \\ aniversidad Peruana de Ciencias Aplicadas. Lima, Perú. \\ ${ }^{2}$ Departmento de Ginecología y Obstetricia, Hospital Nacional Edgardo Rebagliati Martins, Lima, Perú. \\ ${ }^{\text {b}}$ Universidad Nacional Mayor de San Marcos. Lima, Perú.
}

\begin{abstract}
Correspondencia
Omar Yanque

omaryanque@gmail.com

Teléf. +51991215280

Direcc. Hospital Nacional Edgardo

Rebagliati Martins, Av. Edgardo

Rebagliati 490, Jesús María, Lima, Perú.
\end{abstract}

Recibido: 17 de agosto 2017.

Aceptado: 9 de septiembre 2017

Conflictos de interés: Ninguno

declarado.

Financiamiento: Autofinanciado.

Citar como: Cassana A, Yanque $O$. Embarazo en cicatriz de cesárea previa: ¿Es siempre quirúrgico? Reporte de un caso. An Fac med. 2017;78(4):430-4

DOI: http://dx.doi.org/10.15381/

anales.v78i4.14266

\section{An Fac med. 2017;78(4):430-4 / http://dx.doi.org/10.15381/anales.v78i4.14266}

\section{Resumen}

El embarazo en cicatriz de cesárea previa (CSP) es un tipo raro de embarazo ectópico que debe ser diagnosticado tempranamente para poder encontrar la mejor opción terapéutica. Presentamos un caso de CSP, inicialmente mal diagnosticado como una gestación no evolutiva que fue sometida a legrado uterino. La paciente fue referida a nuestra institución, donde el manejo médico conservador con metotrexato falló, y la única opción fue la resección quirúrgica del CSP. El CSP debe de ser sospechado y diagnosticado lo más temprano posible. En países en vías de desarrollo, se debe priorizar la prevención de hemorragia masiva asi como de morbilidad materna compleja. El abordaje mínimamente invasivo debe de ser planificado como el tratamiento de primera línea sobre el manejo médico.

Palabras clave: Embarazo ectópico; Hemorragia uterina; Laparotomía.

\section{Abstract}

Cesarean scar pregnancy (CSP) is a rare type of ectopic pregnancy that should be diagnosed early in order to seek the best therapeutic option. We present a case of CSP initially misdiagnosed as a non-viable pregnancy that undergone curettage. Patient was referred to our institution where conservative medical management with methotrexate failed, and the only option was surgical resection of CSP. CSP should be suspected and diagnosed as early as possible. In low-income countries the prevention of massive blood loss along with serious maternal morbidity should be priorized. The use of minimally invasive approach should be planned as a first-line treatment over medical management.

Keywords: Ectopic pregnancy; Uterine hemorrhage; Laparotomy. 


\section{INTRODUCCIÓN}

Los últimos 10 años han visto la acumulación de gran cantidad de información acerca de los embarazos ectópicos, especialmente su epidemiología, factores de riesgo, y diagnóstico. Sin embargo, el tratamiento de las pacientes con estos embarazos permanece siendo un tópico de debate; aún no existen guías ni consenso para clarificar la elección entre los diferentes tratamientos. En países en vías de desarrollo, como el nuestro, el embarazo ectópico es una condición que potencialmente amenaza la vida, no solo por la enfermedad per se, sino también, y más importante, por las dificultades en reconocer formas raras de presentación (1).

El embarazo en cicatriz de cesárea previa (CSP) es un tipo raro de embarazo ectópico donde las células del trofoblasto se implantan en el nicho de la cicatriz, una condición que potencialmente amenaza la vida que, si no es detectada tempranamente y manejada agresivamente, puede resultar en ruptura uterina, hemorragia, coagulación intravascular diseminada, y muerte materna. La incidencia reportada de CSP oscila entre 1/1800 a 1/2216 embarazos, representando el 0,15\% de todos los embarazos ectópicos, y cerca del $6 \%$ de todos los embarazos ectópicos en mujeres con al menos un parto por cesárea ${ }^{(2,3,4)}$. Aunque es probable que la incidencia se incremente sustancialmente en el futuro cercano a medida que las tasas de parto por cesárea continúan en ascenso ${ }^{(1,2,4)}$.

Muchas veces el CSP no tiene síntomas específicos y puede ser fácilmente mal diagnosticado ${ }^{(2,3)}$. Esta particularidad puede llevar a consecuencias que amenazan la vida, descritas anteriormente. Por lo tanto, el diagnóstico temprano y preciso es importante para un tratamiento efectivo, pudiendo prevenir complicaciones catastróficas ${ }^{(5,6,7)}$.

A la fecha, varias intervenciones han sido propuestas, pero no existe un consenso sobre el protocolo terapéutico óptimo para el CSP. El enfoque depende de múltiples factores tales como la edad gestacional, estabilidad hemodinámica, disponibilidad de experticia endoscópica, fertilidad futura y factibilidad de se- guimiento seriado mediante serología e imágenes ${ }^{(3,5)}$.

Presentamos un caso de CSP identificado luego de un legrado uterino por gestación no evolutiva, que fue referido a nuestra institución por hemorragia masiva transvaginal. Su presentación clínica, diagnóstico, y manejo son discutidos junto con una revisión de la literatura publicada sobre el tema. El objetivo principal del reporte es enfatizar la necesidad de un diagnóstico temprano y las dificultades del manejo médico conservador, terminando en un tratamiento quirúrgico abierto. Se incluyen recomendaciones diagnósticas y de manejo al reporte.

\section{CASO CLÍNICO}

Una paciente mujer de 23 años de edad, grávida 2 para 1 (G2P1011, parto por cesárea), fue referida del Hospital de Iquitos (1 011 km de Lima, Perú) a nuestro centro en el tercer día (PO3) luego de un legrado uterino, buscando la confirmación de un embarazo ectópico cervical. Su edad gestacional fue de 13 2/7 semanas al momento de su admisión en nuestro
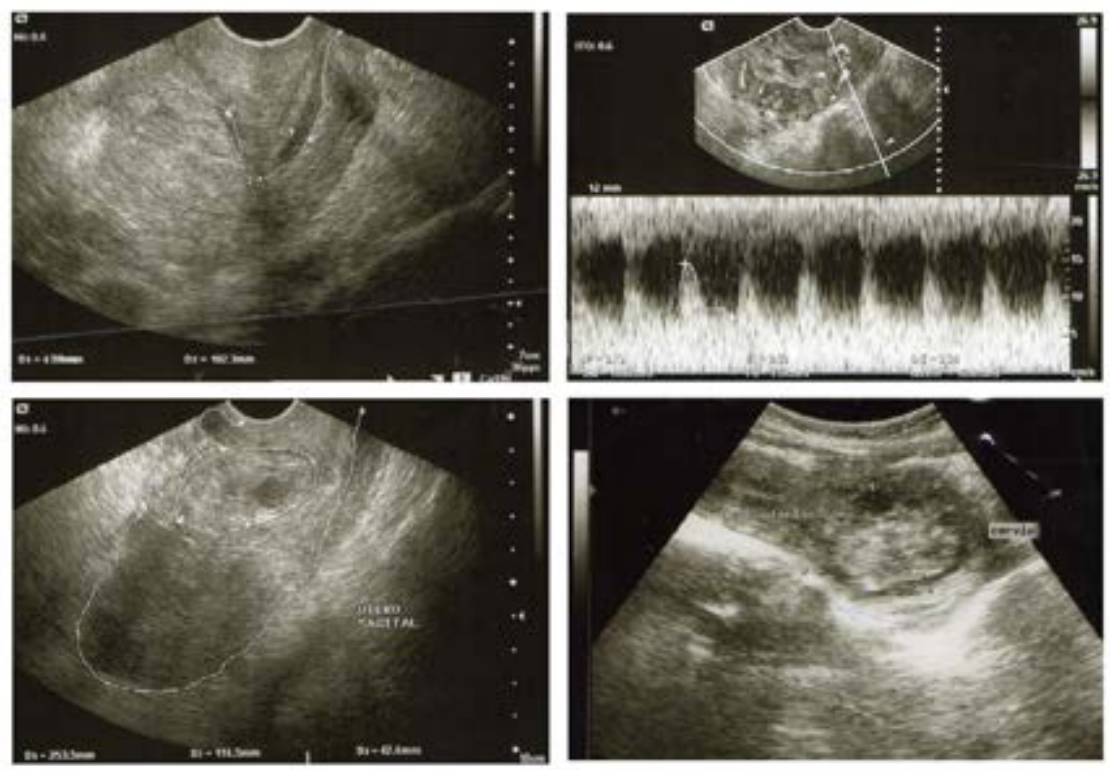

Figura 1. (Superior e inferior izquierda) Vista sagital de la ecografia transvaginal mostrando una masa irregular con ecos mixtos de $65 \times 51 \times 41 \mathrm{~mm}$ al nivel del istmo uterino. (Superior derecha)

Doppler color de la masa irregular. (Inferior derecha) Vista sagital de la ecografía pélvica mostrando el fondo uterino a la izquierda y el cérvix a la derecha, con una masa irregular al medio. hospital. La paciente tenía una historia de dolor pélvico súbito que motivó su visita ticada con una gestación no evolutiva, realizándose un legrado uterino. Durante el procedimiento, una hemorragia masiva hizo detener al cirujano, colocando un tapón vaginal y suspendiendo el acto quirúrgico. Dos días luego del procedimiento, una ecografía transvaginal mostró la posibilidad de un embarazo ectópico cervical, por lo que la paciente fue referida a nuestra institución, en Lima.

Al momento de la admisión, se evidenció un cérvix cerrado al examen pélvico con un útero de $12 \mathrm{~cm}$, doloroso al examen digital, una pequeña cantidad de sangre oscura en la cavidad vaginal, sin sangrado activo. El resto de sistemas sin hallazgos significativos. La ecografía transvaginal mostraba un útero de $108 \mathrm{x}$ $38 \times 41 \mathrm{~mm}$ con bordes irregulares, una cavidad uterina vacía, y una masa de ecos mixtos de $65 \times 51 \mathrm{~mm}$, localizada al nivel del istmo uterino, con vascularización periférica y central (Figura 1). No se observaron masas anexiales, ni líquido libre en cavidad. al Hospital de lquitos, donde fue diagnos- 


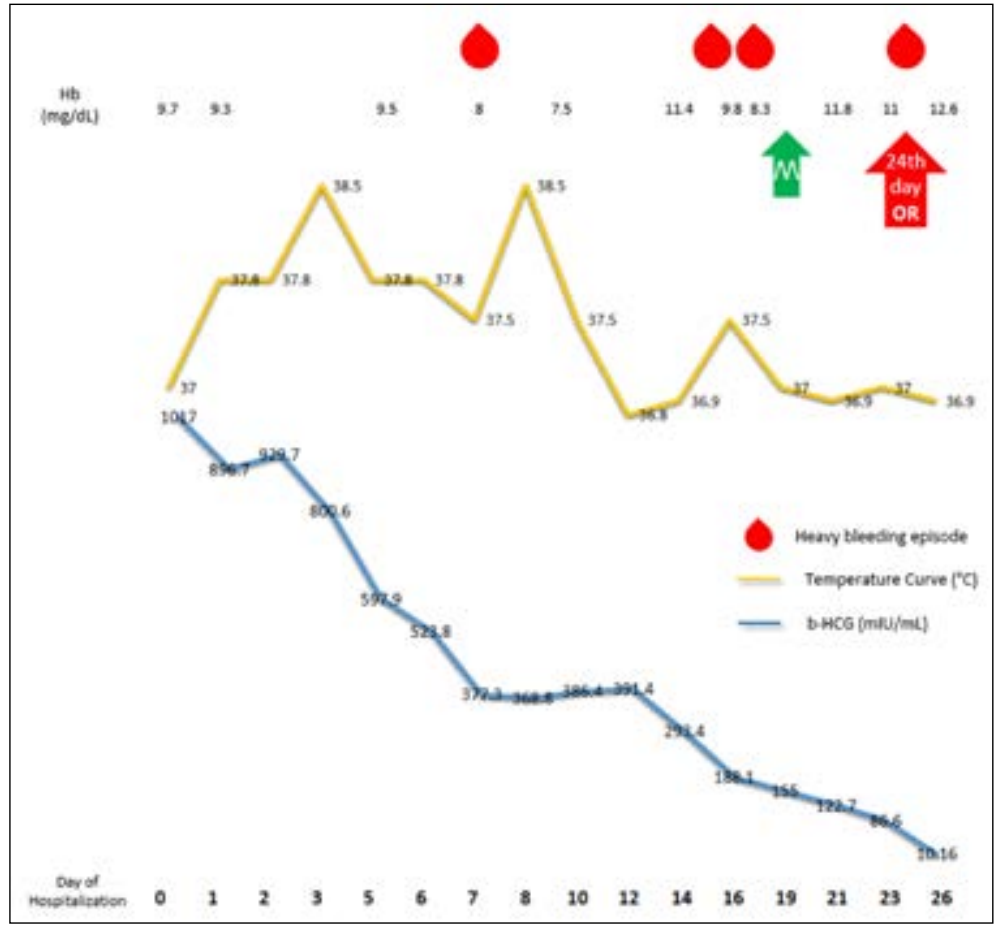

Figura 2. Resumen de la evolución de la paciente, mostrando niveles de ßhCG, temperatura, y episodios de hemorragia transvaginal. La flecha verde indica el día de terapia con metotrexato. La flecha roja indica el día de la laparotomía.

Fue admitida a nuestra unidad con un nivel de BhCG de $1017 \mathrm{mUl} / \mathrm{ml}, \mathrm{Hb}$ $9,7 \mathrm{~g} / \mathrm{dl}$, Leucocitos 7040 células $/ \mathrm{mm} 3$ y el diagnóstico de embarazo en cicatriz de cesárea previa (CSP). Fue manejada inicialmente con antibióticos por la alta sospecha de endometritis, con una temperatura máxima de $38,5^{\circ} \mathrm{C}$ sin respuesta clínica luego de 5 días, con valores de $\beta$ hCG en tendencia descendente, sin sangrado transvaginal (Figura 2). Se realizaron revisiones ecográficas cada 48 horas.

Se tomó la decisión de usar prostaglandinas para evacuar cualquier contenido endouterino y suavizar el cérvix para un futuro legrado con visión ecográfica. Luego de 3 dosis $(200 \mu \mathrm{g} /$ intravaginal cada una) se evidenció una hemorragia abundante, estabilizando a la paciente con cristaloides y una unidad de sangre (paquete globular). Luego de 2 días de este episodio, una hemoglobina control fue obtenida con un valor de $7,5 \mathrm{~g} / \mathrm{dL}$. Se instauró un nuevo antibiótico y transfundieron dos unidades de sangre más. Luego de una semana la paciente tuvo dos episodios más de hemorragia trans-
Cuatro meses después del alta, se realizó una ecografía transvaginal a la paciente, siendo informada como útero y anexos sin alteraciones. Exámenes clínicos realizados 4 y 6 meses posteriores al alta fueron normales. 15 meses luego del alta, la paciente embarazó y dio a luz a un bebé de 3090 gramos a las 38 semanas por cesárea en una clínica particular.

\section{DISCUSIÓN}

Se conoce poco acerca de la fisiopatología exacta del embarazo en cicatriz de cesárea previa. Los factores predisponentes más discutidos son la migración acelerada del blastocisto a través de la cavidad uterina, las condiciones desfavorables para la implantación en el endometrio, el daño del canal cervical, endometrio a nivel de la cicatriz de cesárea previa que no cicla al unísono con el resto de endometrio de la cavidad uterina, entre otros factores ${ }^{(8,9)}$. El mecanismo más probable que puede explicar la implantación a nivel de la cicatriz es que existe invasión del miometrio a través del tracto microtubular entre la cicatriz de cesárea previa y el canal endometrial. Dicho tracto puede ser también desarrollado de un trauma (noxa) de otra cirugía uterina, como legrado uterino, miomectomía, metroplastía, histeroscopía e inclusive remoción manual de la placenta ${ }^{(5)}$. Timor-Tritsch y col., mostraron estudios in vitro donde el tejido cicatricial favorecería un ambiente de tensión baja de oxígeno, estimulando al citotrofoblasto a invadir profundamente el área cicatricial. También se ha demostrado, en otro estudio in vitro, la fuerte propensión del trofoblasto a adherirse a la matriz extracelular expuesta, en
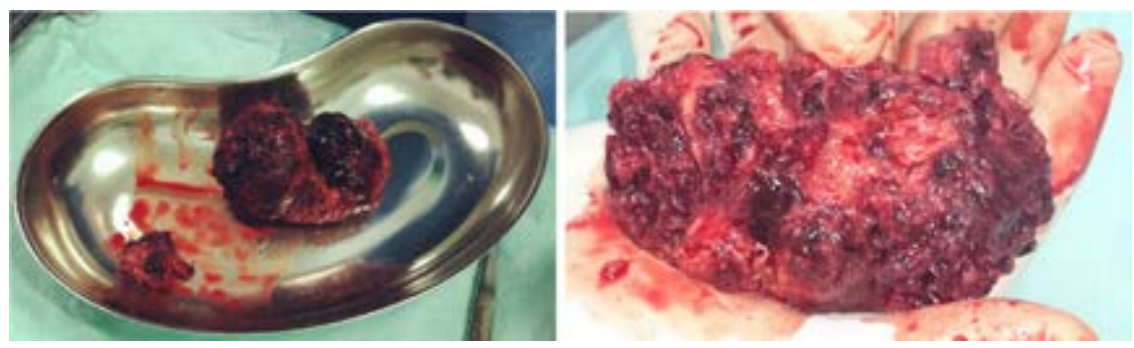

Figura 3. Masa del embarazo en cicatriz de cesárea previa 


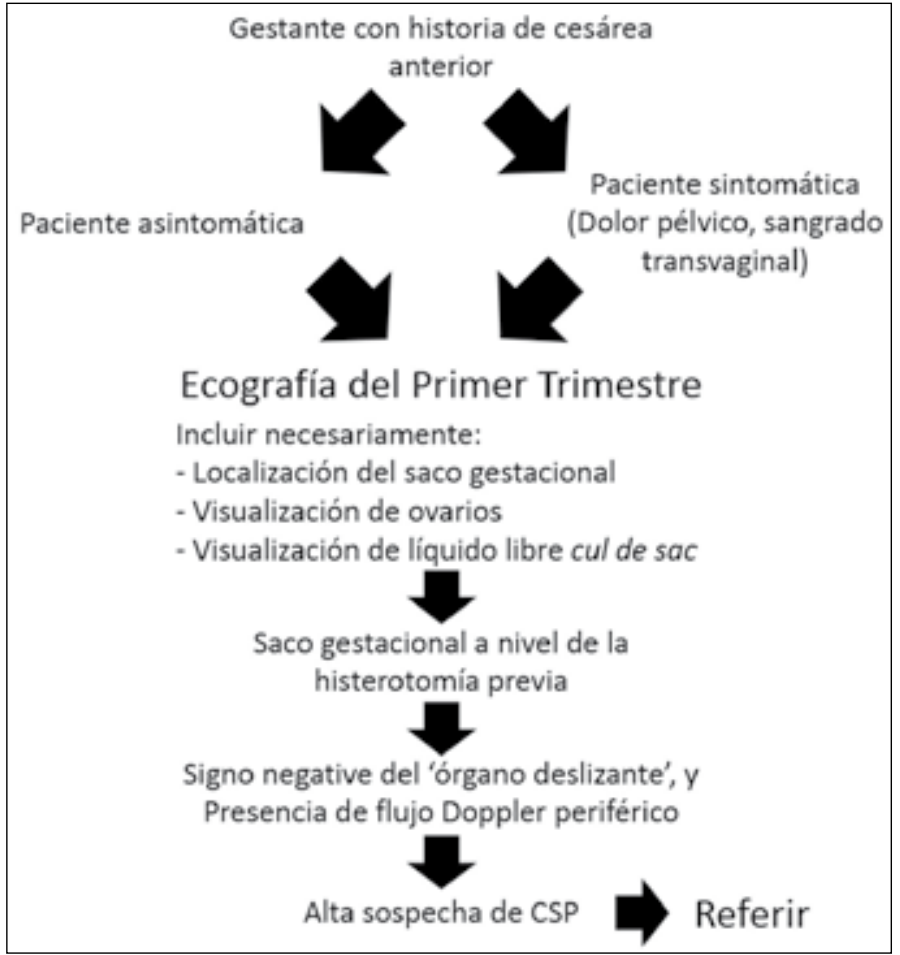

Figura 4. Algoritmo propuesto para referencia a hospitales del tercer nivel de atención.

vez de a las células epiteliales endometriales ${ }^{(10)}$. Por consiguiente, se necesitan más investigaciones en la patogénesis de esta entidad para poder explicar el por qué algunas pacientes van a desarrollar un embarazo en la cicatriz de cesárea previa, y si el CSP y la placenta acreta representan diferentes estadios de una enfermedad continua que lleva a una placenta mórbidamente adherida en el tercer trimestre ${ }^{(11)}$.

Ya que el examen clínico en las pacientes estables no muestra peculiaridad alguna, la piedra angular del diagnóstico es una ecografía transvaginal en las primeras semanas del embarazo $(1,5)$. Existen criterios diagnósticos ya establecidos para el diagnóstico de un embarazo en cicatriz previa de cesárea ${ }^{(5)}$. Zhou y col., mostraron que tanto la ecografía transabdominal y la transvaginal juntas, pueden mejorar la tasa de detección del sitio de implantación del huevo fecundado, y de esta manera proveer el soporte imagenológico para iniciar un programa terapéutico, mejorando la tasa de detección de cualquier embarazo ectópico en general ${ }^{12)}$. Los criterios diagnósticos para CSP por ultrasonido deben de incluir la visualización del saco gestacional en el sitio de la histerotomía previa, fuera de la cavidad endometrial. Un miometrio delgado o ausente entre el saco gestacional y la vejiga. Un signo negativo de 'deslizamiento de órgano' (traducido del inglés 'sliding organ') y la presencia de flujo Doppler periférico son también esperados en la evaluación ${ }^{(2,3,12)}$.

EI CSP no debe ser manejado de manera expectante, aun cuando la historia natural de algunos CSP puede ser naturalmente un aborto. Liu y col., reportaron y revisaron el manejo expectante del CSP, mostrando que la literatura describía que más del $50 \%$ de casos reportaban ruptura uterina, hemorragia masiva $y$ complicaciones que amenazaban la vida de la paciente. Es por ello, que la terapia médica o quirúrgica debe de ser considerada antes de la ruptura para remover el saco gestacional, con una disminución en la morbilidad materna extrema y con el objetivo de preservar la fertilidad futura de la paciente ${ }^{(13,14,16)}$.

Litwicka y col., resumieron las distintas modalidades de tratamiento para el CSP, desde el tratamiento médico con diferentes regímenes farmacológicos al tratamiento quirúrgico con enfoques mínimamente invasivos o convencionales, y una combinación de ambos. Concluyeron que el tratamiento del CSP conlleva una tasa importante de complicaciones. El mayor número de complicaciones se observó en las estrategias tales como el metotrexato sistémico y el legrado uterino. Por otro lado, las tasas más bajas de complicaciones fueron alcanzadas mediante el uso de inyección intragestacional de metotrexato o cloruro de potasio, así como con la histeroscopía ${ }^{(18)}$. La inyección intragestacional de metotrexato guiada por ecografía transvaginal minimiza su absorción sistémica, maximizando su acción local, degenerando de esta manera las células de las vellosidades coriónicas más rápidamente. Otros embriocidas, tales como el cloruro de potasio o el etanol, pueden ser usados alcanzando resultados comparables con el metotrexato ${ }^{(18,19)}$. Birch Petersen y col. en una revisión sistemática de los estudios de tratamiento escogieron condensar y simplificar las modalidades de tratamiento en 14 enfoques, demostrando que la literatura apoya un enfoque intervencionista en vez de uno médico ${ }^{(19)}$.

La demora en iniciar el metotrexato en nuestra paciente fue la falta de respuesta clínica al tratamiento de endometritis, una consecuencia del mal diagnóstico inicial. La principal razón para retrasar la intervención quirúrgica fue dar la opción de iniciar el tratamiento con metotrexato, evitando la opción de una histerectomía inicial, según lo deseado por la paciente. Decisiones como ésta no deben ser tomadas en instituciones que no cuenten con equipos multidisciplinarios las 24 horas del día, banco de sangre $y$, disponibilidad de sala de operaciones. En casos de CSP, donde no se cuenta con guías de tratamiento universal, el proceso de toma de decisiones siempre debe de ser en conjunto con la paciente, explicando en detalle todos los pros y contras del manejo propuesto.

En este caso, y de otros pocos casos no reportados de CSP en nuestro hospital, el tratamiento médico de esta condición no fue efectivo, y puede llevar a un riesgo innecesario para la paciente 


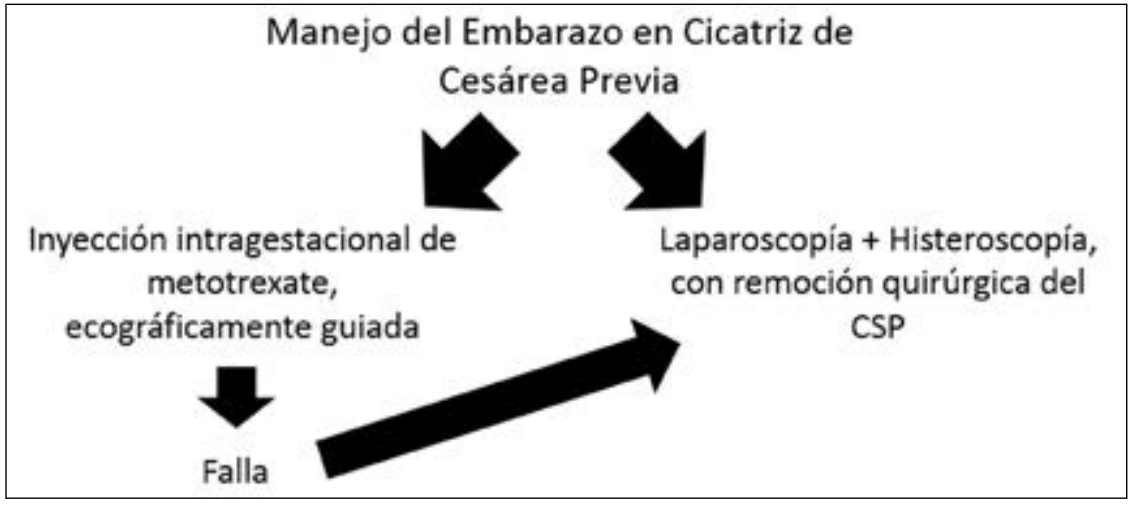

Figura 5. Algoritmo propuesto simplificado para el manejo de CSP.

en términos de complicaciones e histerectomía. En algunas realidades, como la nuestra, la embolización de arterias uterinas no es la primera opción debido a la falta de experticia en el área, así como la falta de recursos ${ }^{(17)}$. El manejo quirúrgico debe de ser planificado como el tratamiento de primera línea con un enfoque mínimamente invasivo para asegurar la fertilidad y disminuir la posibilidad de una histerectomía ${ }^{(15)}$. El legrado uterino, por sí solo, no debe ser una opción inicial, y la inyección intragestacional de metotrexato debe de ser incentivada en escenarios como los nuestros. Adicionalmente, los resultados reproductivos luego de un CSP deben de ser explicados a la paciente y la familia, a fin de dar una consejería adecuada, enfatizando los riesgos de un nuevo CSP, acretismo placentario o una cesárea-histerectomía en embarazos posteriores ${ }^{(20)}$.

La sospecha o diagnóstico de CSP (Figura 4) debe de ser suficiente para referir a la paciente a instalaciones médicas de mayor resolución, a fin de disminuir la chance de morbilidad materna extrema o seria, y la muerte. El entrenamiento en ultrasonografía para patologías complejas, usualmente raras, debe ser reforzado en escenarios rurales con cierta regularidad. Un algoritmo (Figura 5) que incluye el manejo quirúrgico como el tratamiento de primera línea en hospitales de referencia, debe de ser tomado en consideración, con énfasis en técnicas mínimamente invasivas. Mayores datos locales son necesarios para dar al tratamiento médico algún rol en el manejo de esta patología.
El embarazo en cicatriz de cesárea previa (CSP) es una condición que potencialmente amenaza la vida, debe de ser sospechada y diagnosticada lo más temprano posible. En países en vías de desarrollo, los objetivos principales en el tratamiento del CSP son la prevención de hemorragia masiva junto con morbilidad materna extrema o seria, y por último la conservación del útero para mantener la fertilidad futura, la salud de la mujer y la calidad de vida. Un enfoque mínimamente invasivo debe de ser planificado como el tratamiento de primera línea en escenarios como los nuestros, donde tecnolodisponible.

\section{REFERENCIAS BIBLIOGRÁFICAS}

1. Awad E, El-agwany A, El-habashy A, Elmansy A. Lower uterine segment pregnancy (Cesarean Scar Pregnancy and early placenta accreta): A rising complication from cesarean section with possible and similar early ultrasound diagnoses and management. The Egyptian Journal of Radiology and Nuclear Medicine. 2015;46(4):977-980. DOI: 10.1016/j.ejrnm.2015.05.018

2. Panelli DM, Phillips $\mathrm{CH}$, Brady PC. Incidence, diagnosis and management of tubal and nontubal ectopic pregnancies: a review. Fertility Research and Practice. 2015;1(15):1-20. DOI: 10.1186/ s40738-015-0008-z

3. Patel MA. Scar Ectopic Pregnancy. J Obstet Gynaecol India. 2015;65(6):372-5. DOI: 10.1007/ s13224-015-0817-3

4. Phupong $V$, Narasethkamol A, Ultchaswadi P. Pregnancy in caesarean section scar. J Obstet Gynaecol. 2011;31(3):204-6. DOI: 10.3109/01443615.2010.546907

5. Ash A, Smith A, Maxwell D. Caesarean scar pregnancy. BJOG. 2007;114(3):253-63. DOI: 10.1111/j.1471-0528.2006.01237.x

6. Riaz RM, Williams TR, Craig BM, Myers DT. Cegía más nueva y más segura aún no está sarean scar ectopic pregnancy: imaging features, current treatment options, and clinical outcomes. Abdom Imaging 2015;40(7):2589-99. DOI: 10.1007/ s00261-015-0472-2

7. Sadeghi H, Rutherford T, Rackow BW, Campbell $\mathrm{KH}$, Duzyj CM, Guess MK, et al. Cesarean Scar Ectopic Pregnancy: Case Series and Review of the Literature. Am J Perinatol. 2010;27(2):111-20. DOI: 10.1055/s-0029-1224874

8. Ben-Nagi J, Walker A, Jurkovic D, Yazbek J, Aplin JD. Effect of cesarean delivery on the endometrium. Int J Gynaecol Obstet. 2009;106(1):30-4. DOI: 10.1016/j.ijgo.2009.02.019

9. Tomov S, Gortchev G, Tantchev L, Nikovola M, Popovska S. Cervical Ectopic Pregnancy With Massive Bleeding: A Case Report. International Journal of Surgery and Medicine. 2015;1(1):22-25. DOI: 10.5455/ijsm.20150511101303

10. Timor-Tritsch IE, Monteagudo A. Unforeseen consequences of the increasing rate of cesarean deliveries: early placenta accreta and cesarean scar pregnancy. A review. Am J Obstet Gynecol. 2012;207(1):14-29. DOI: 10.1016/j.ajog.2012.03.007

11. Timor-Tritsch IE, Monteagudo A, Cali G, PalaciosJaraquemada JM, Maymon R, Arslan AA, et al. Cesarean scar pregnancy and early placenta accreta share common histology. Ultrasound Obstet Gynecol. 2014;43(4):383-95. DOI: 10.1002/uog.13282

12. Jing $Z$, Dan L, Liqing $P$. The characteristics of ectopic pregnancy ultrasound image and value of vaginal ultrasound combined with abdominal ultrasound in diagnosis of ectopic pregnancy. Journal of Hainan Medical University. 2015:161-164.

13. Timor-Tritsch IE, Khatib N, Monteagudo A, Ramos J, Berg R, Kovács S. Cesarean scar pregnancies: experience of 60 cases. J Ultrasound Med. 2015;34(4):601-10. DOI: 10.7863/ultra.34.4.601

14. Sinha P, Mishra M. Caesarean scar pregnancy: A precursor of placenta percreta/accreta. J Obstet Gynaecol. 2012;32(7):621-3. DOI: 10.3109/01443615.2012.698665

15. Wang HY, Zhang J, Li YN, Wei W, Zhang DW, Lu YQ, et al. Laparoscopic Management or Laparoscopy Combined with Transvaginal Management of Type II Cesarean Scar Pregnancy. JSLS. 2013;17(2):26372. DOI: $10.4293 / 108680813 \times 13654754535197$

16. Liu H, Leng J, Shi H, Lang J. Expectant treatment of cesarean scar pregnancy: two case reports and a glimpse at the natural courses. Arch Gynecol Obstet. 2010;282(4):455-8. DOI: 10.1007/s00404010-1391-7

17. Zhang XB, Zhong YC, Chi JC, Shen JL, Qiu $\mathrm{XX}, \mathrm{Xu} J \mathrm{R}$, et al. Caesarean Scar Pregnancy: Treatment with Bilateral Uterine Artery Chemoembolization Combined with Dilation and $\mathrm{Cu}$ rettage. J Int Med Res. 2012;40(5):1919-30. DOI: $10.1177 / 030006051204000533$

18. Litwicka K, Greco E. Caesarean scar pregnancy: a review of management options. Curr Opin Obstet Gynecol. 2013;25(6):456-61. DOI: 10.1097/ gco.0000000000000023

19. Birch Petersen K, Hoffmann E, Rifbjerg Larsen C, Svarre Nielsen H. Cesarean scar pregnancy: a systematic review of treatment studies. Fertil Steril. 2016;105(4):958-67. DOI: 10.1016/j.fertnstert.2015.12.130

20. Gao L, Huang Z, Zhang X, Zhou N, Huang X, Wang $X$. Reproductive outcomes following cesarean scar pregnancy - a case series and review of the literatura. Eur J Obstet Gynecol Reprod Biol. 2016;200:102-7. DOI: 10.1016/j.ejogrb.2016.02.039 